\title{
Selection of Reliable Machine Learning Algorithms for Geophysical Applications
}

C.O. Dumitru, G. Schwarz, D. Ao, G. Dax, V. Andrei, C. Karmakar, and M. Datcu

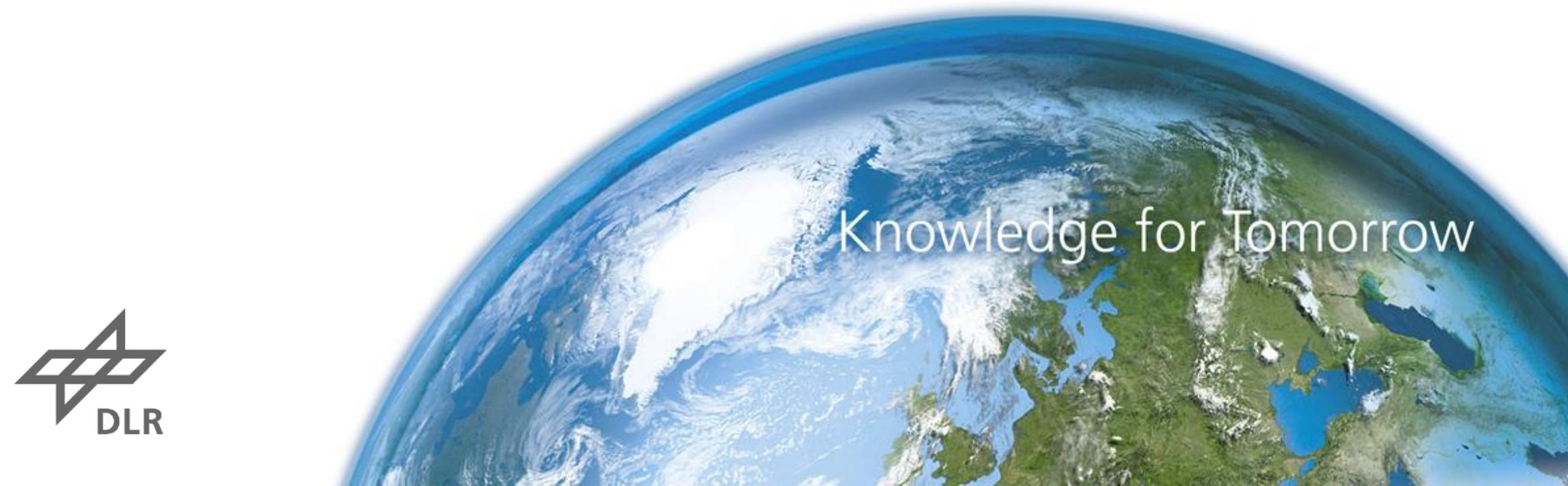




\section{Big Data \& Sensors}

\section{The volume of data for different sensors over the years}
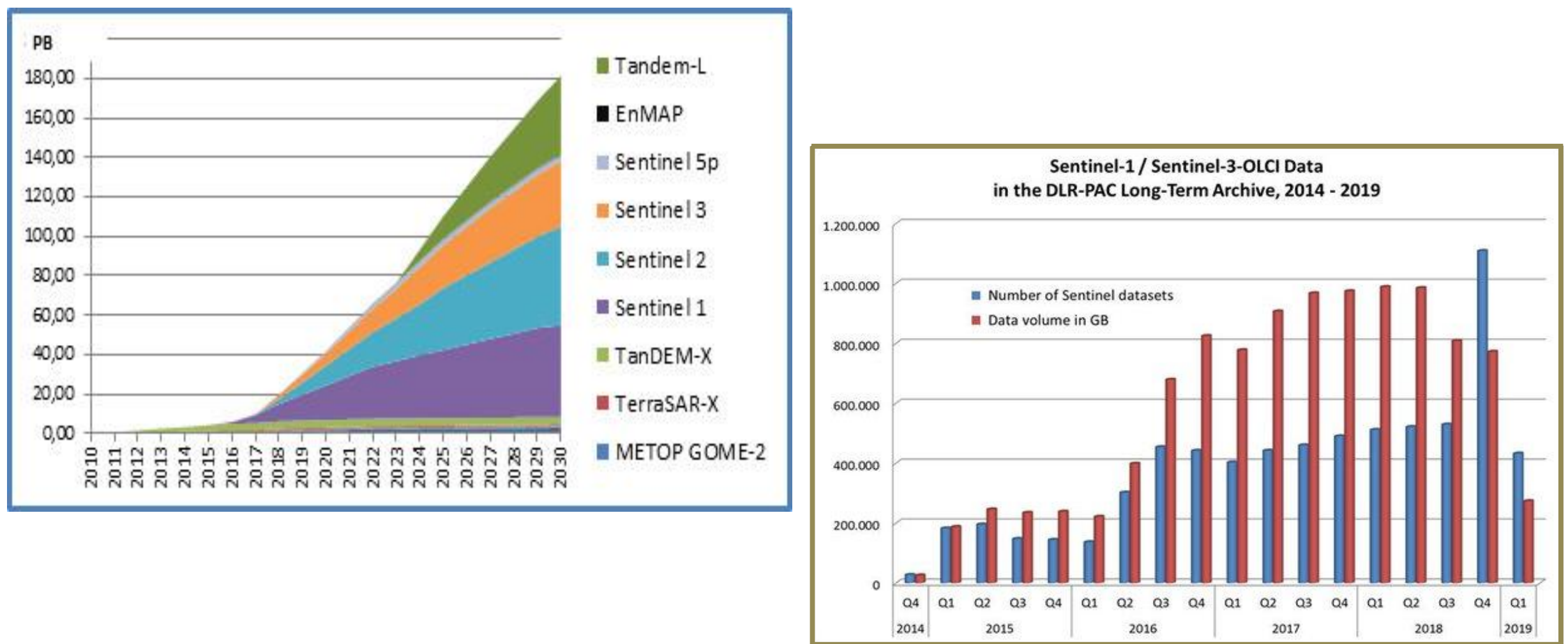

https://www.dlr.de/eoc/en/desktopdefault.aspx/tabid-12632/22039 read-51751

https://www.dlr.de/eoc/en/desktopdefault.aspx/tabid-13247/23165 read-54030

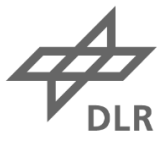




\section{Applications: TerraSAR-X archive semantic catalogue 300 cities 1300 semantic labels}
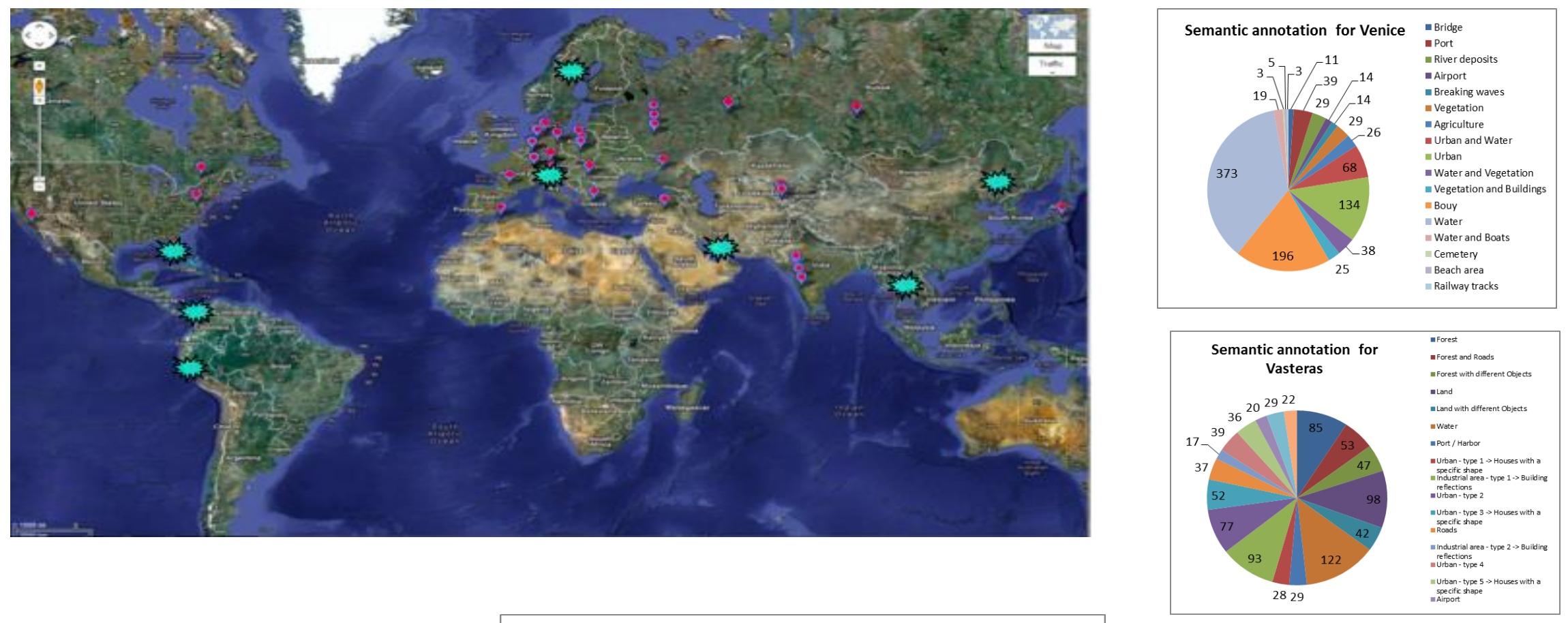

-Bangkok (Thailand); -Shenyang (China); -Nazca Lines (Peru); -Havana (Cuba); -Venice (Italy); -Vasteras (Sweden); -Oran (Algeria); -Bogota (Columbia).

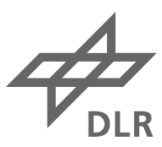

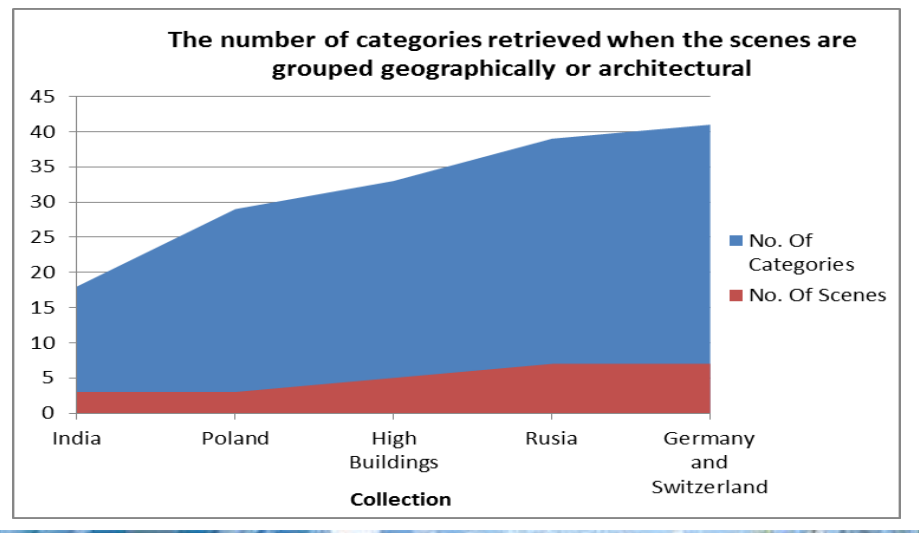

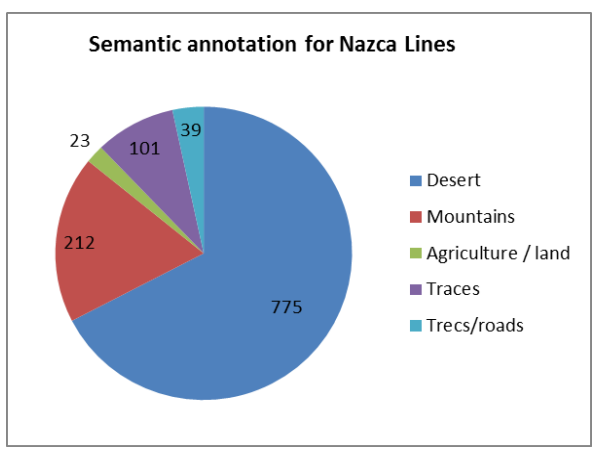




\section{Applications: Protected areas in Europe - ECOPOTENTIAL}
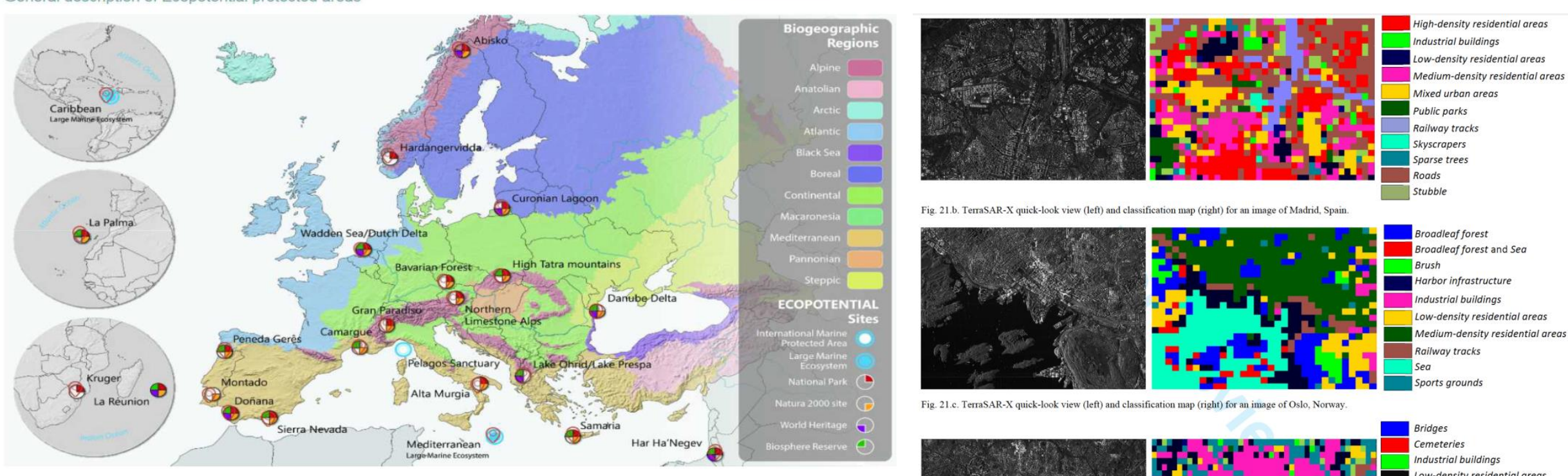

Fig. 21.c. TerraSAR-X quick-look view (left) and classification map (right) for an image of Oslo, Norway.



\section{Data Mining Tools}

\section{Multimodal queries}
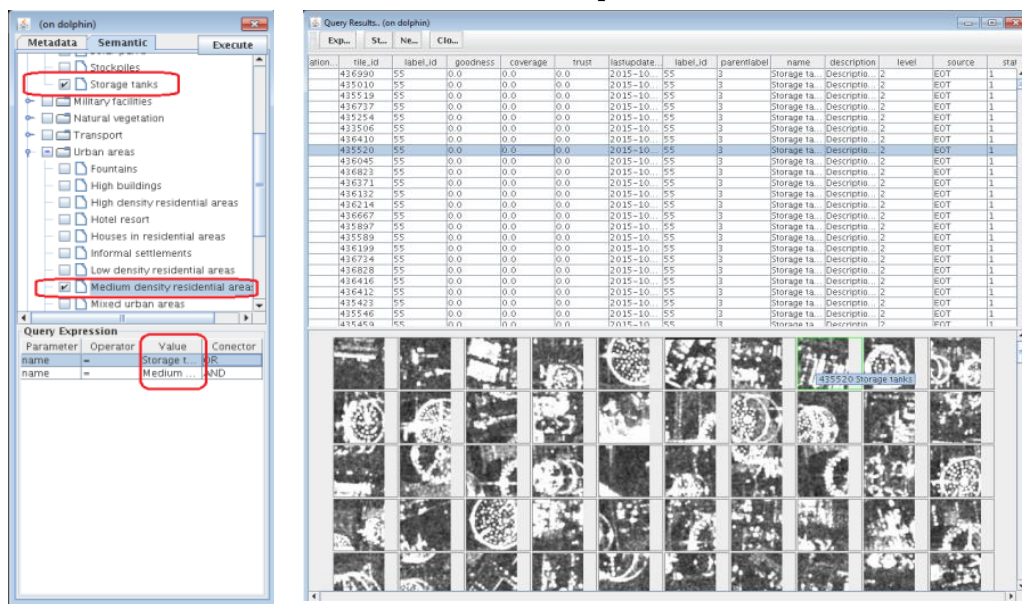

KDD and semantic extraction
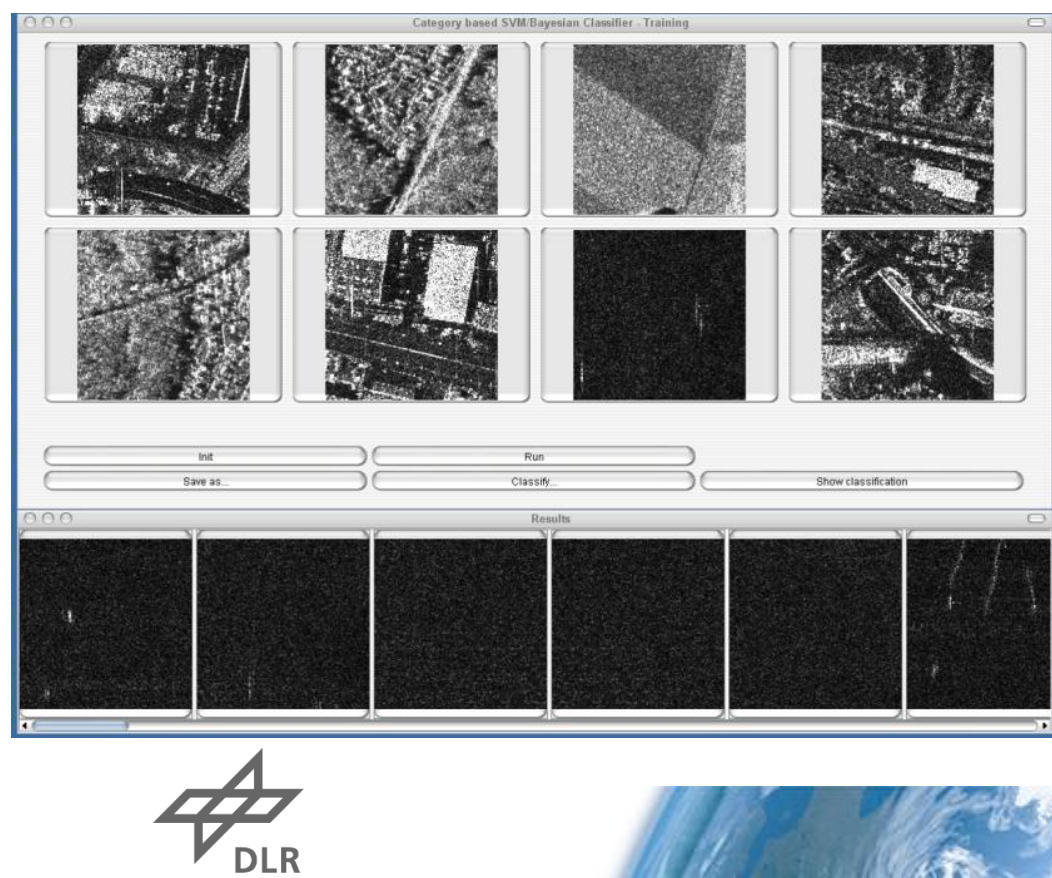

\section{Query by example}

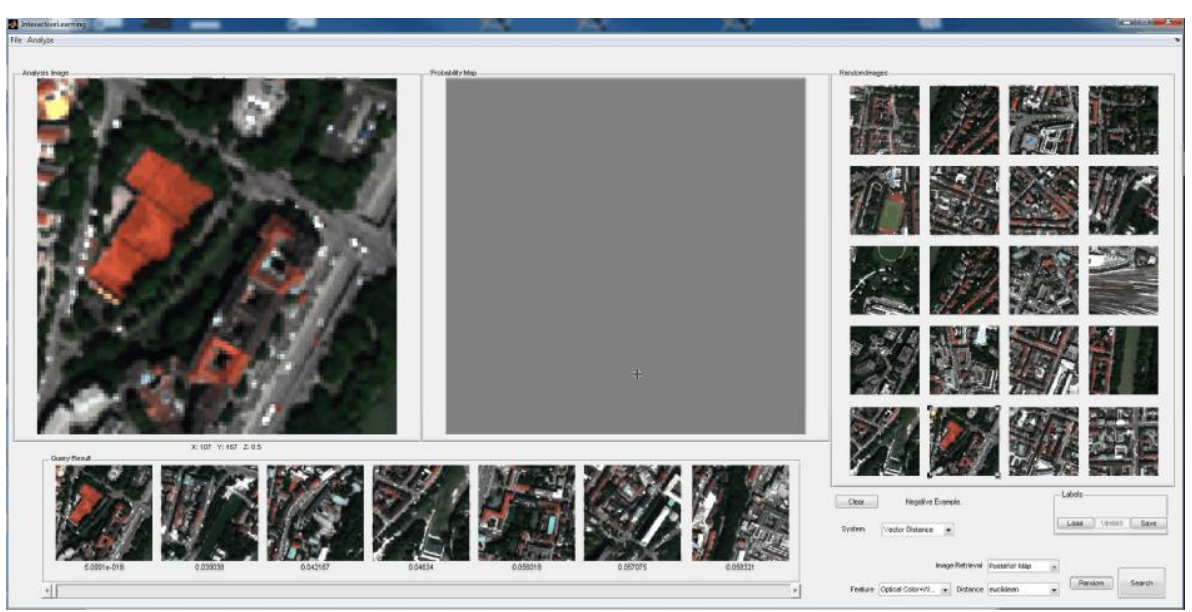

Spatio temporal patterns discovery

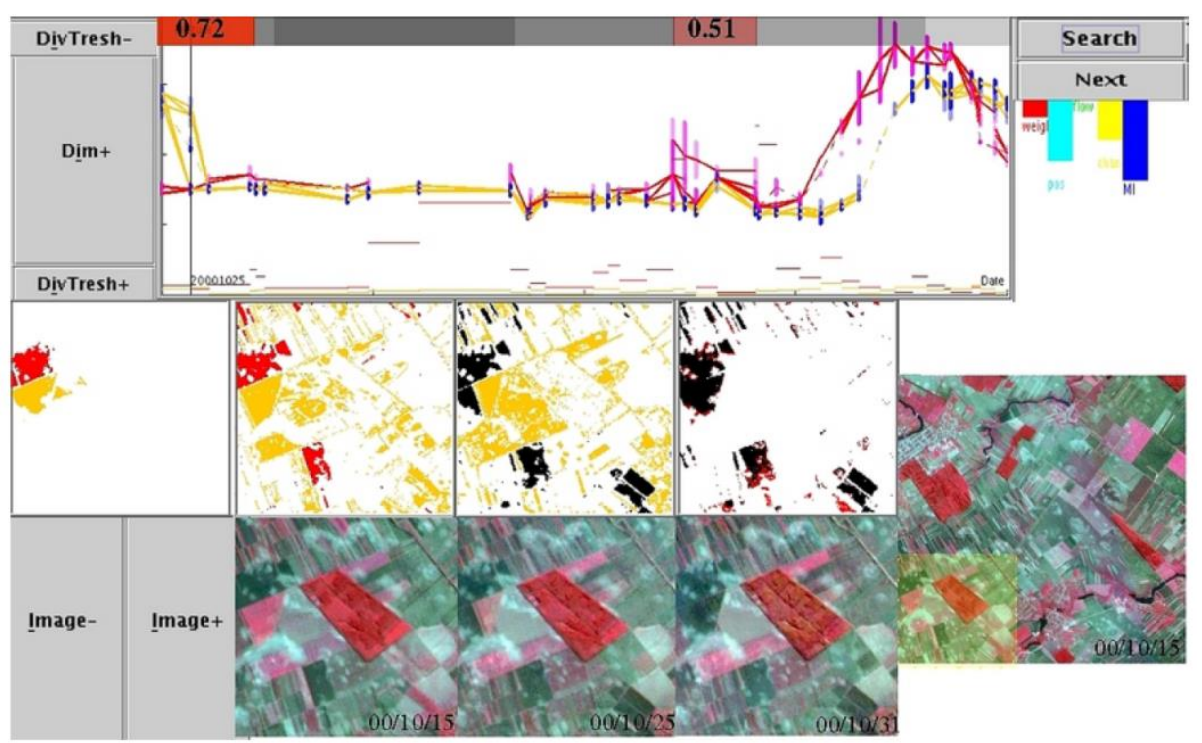




\section{CANDELA}

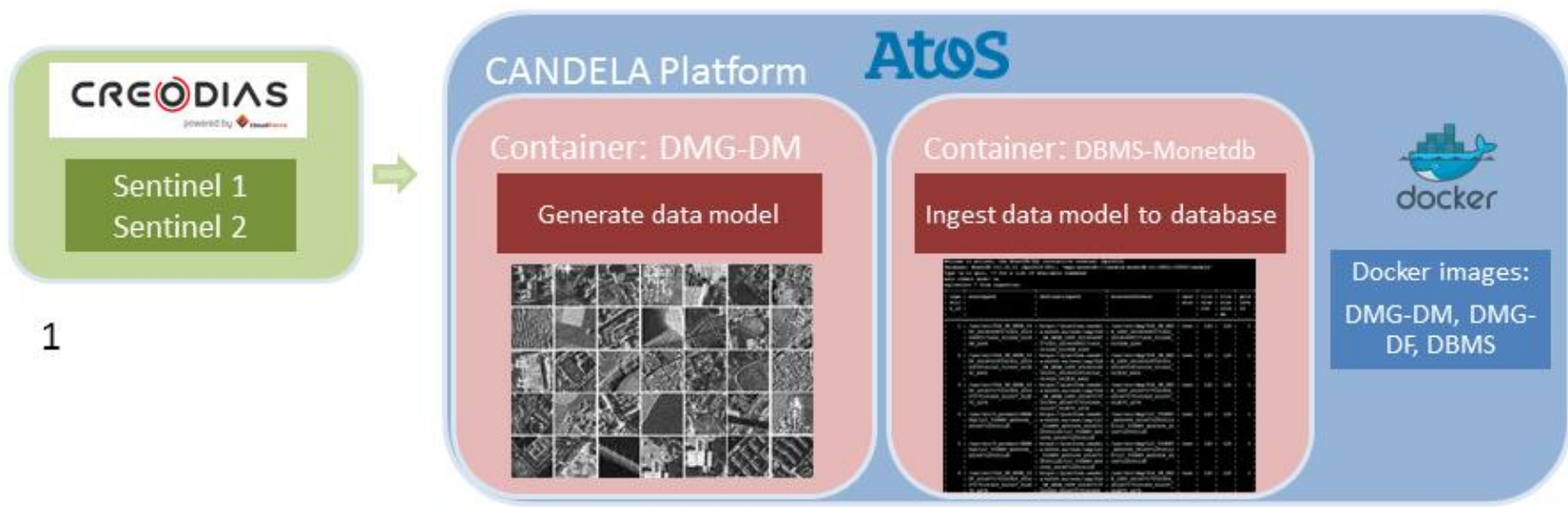

2

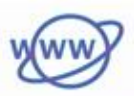

Internet
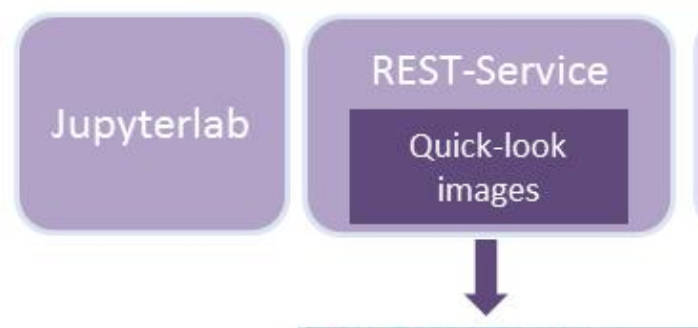

Database in the platform

Query: product metadata

Ingest: annotated semantics

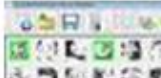
มลำ K: $x \in \cos x$ 2utrases ext a L E E Q स

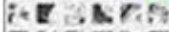

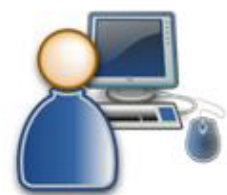

落

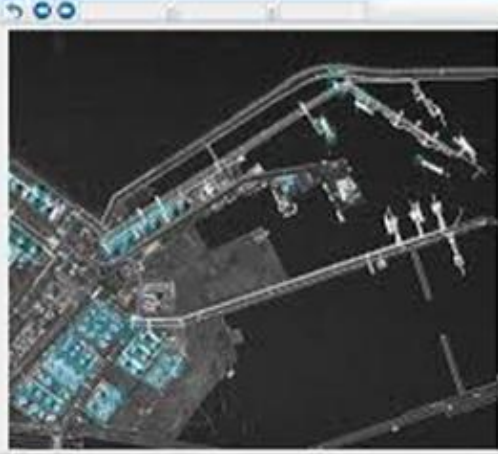

DMG-DM: Data Model Generation for Data Mining DBMS: Database Management System

Within GUI tool:

KDD: Image Search and

Semantic Annotation

QE: Multi-Knowledge and

Query

axis 202

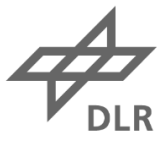




\section{IA for Al: The Information Architecture}

KIM: Bring Data to Algorithms

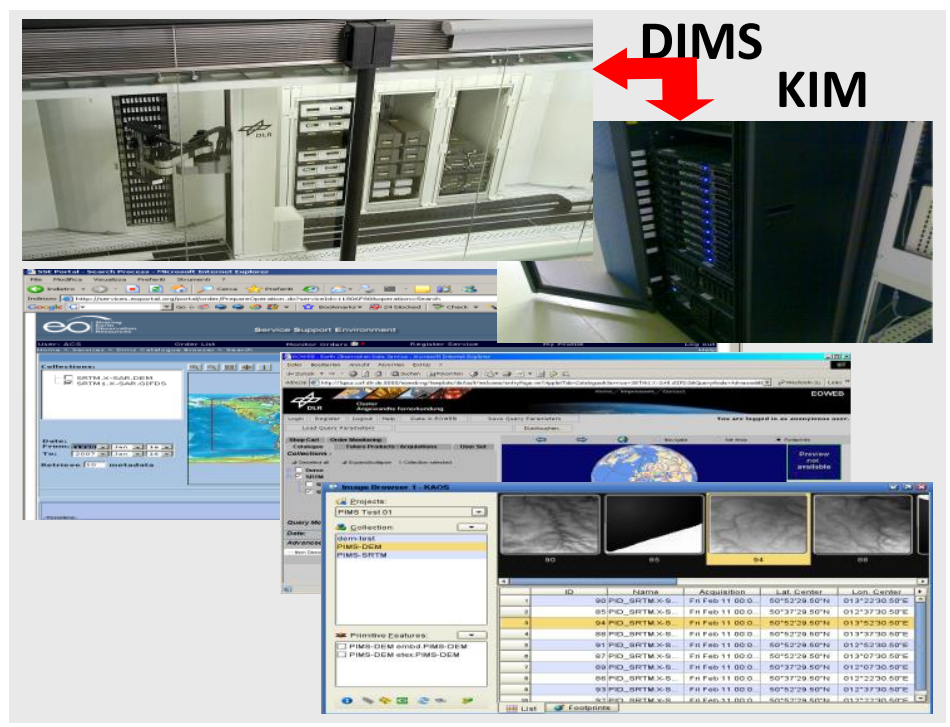

EO Block chain: federated systems

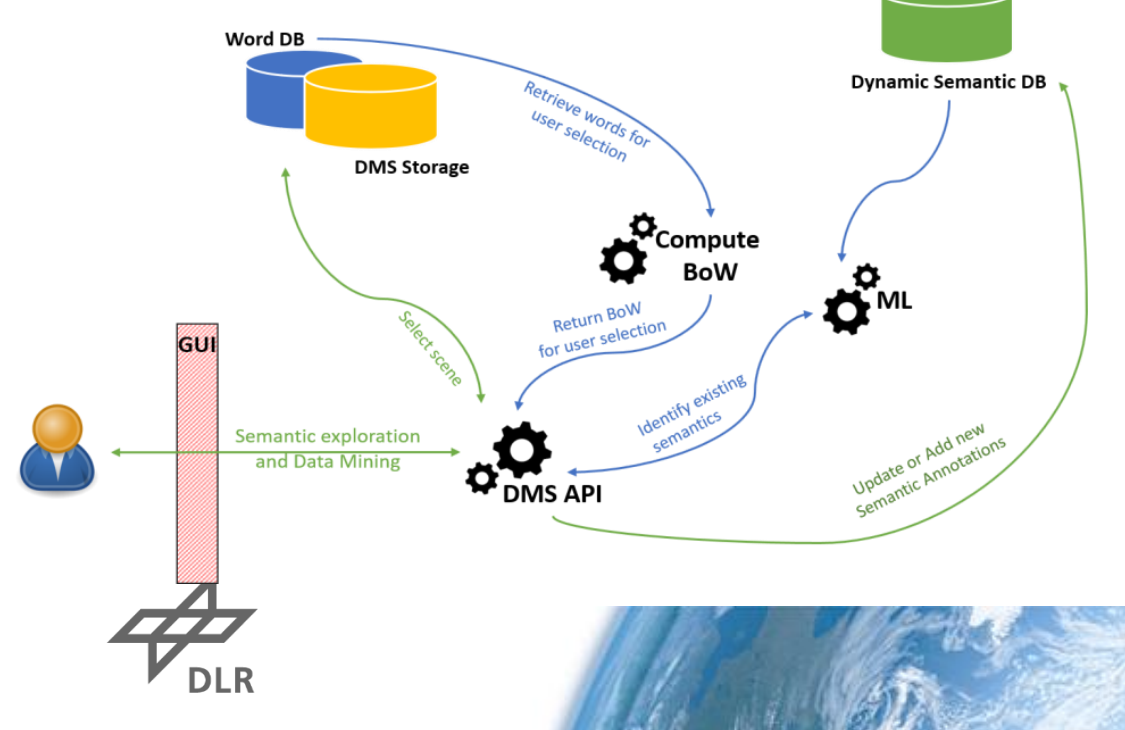

EOLib: Bring Algorithms to Data

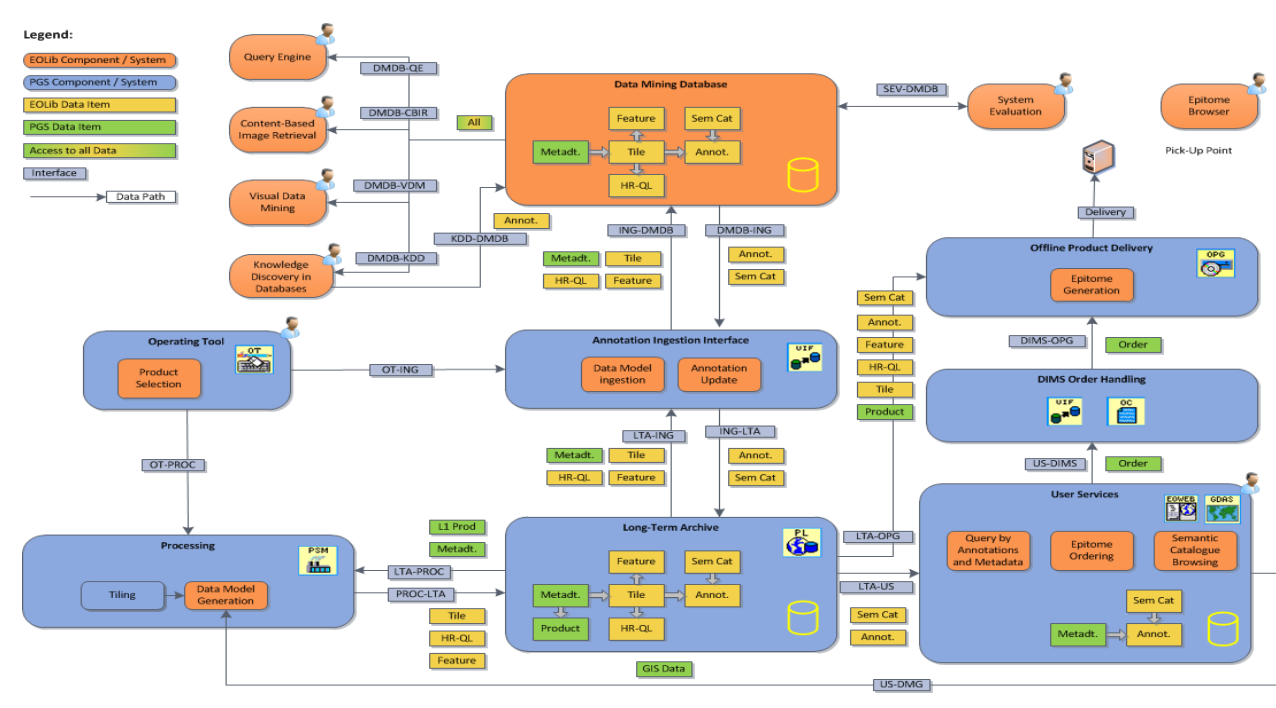

Laser Comm: EO Internet 


\section{Discovery of semantic relationships: PoISAR L-Band}
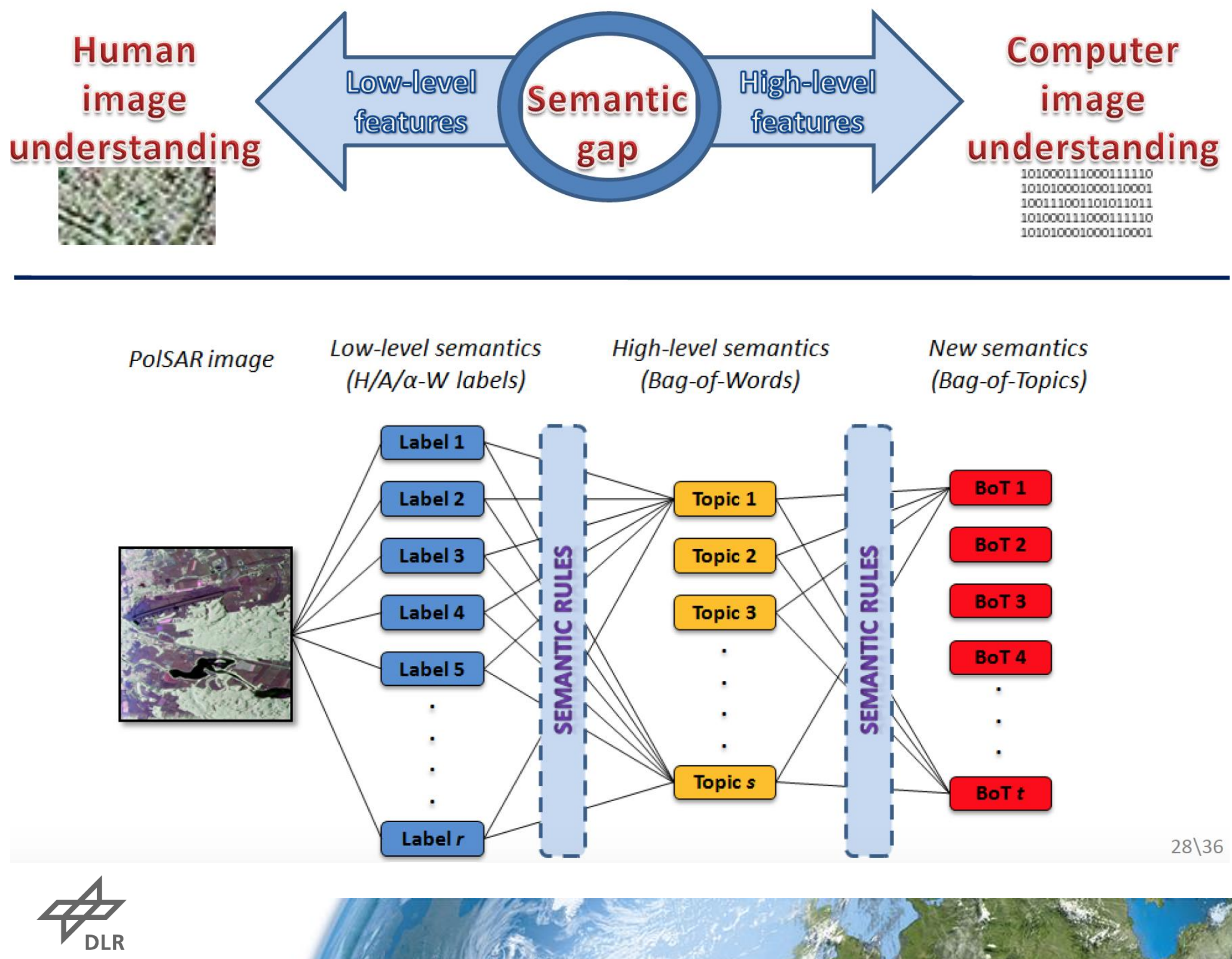


\section{PolSAR L-Band Low-level semantics - H\A| $\alpha$ classes}
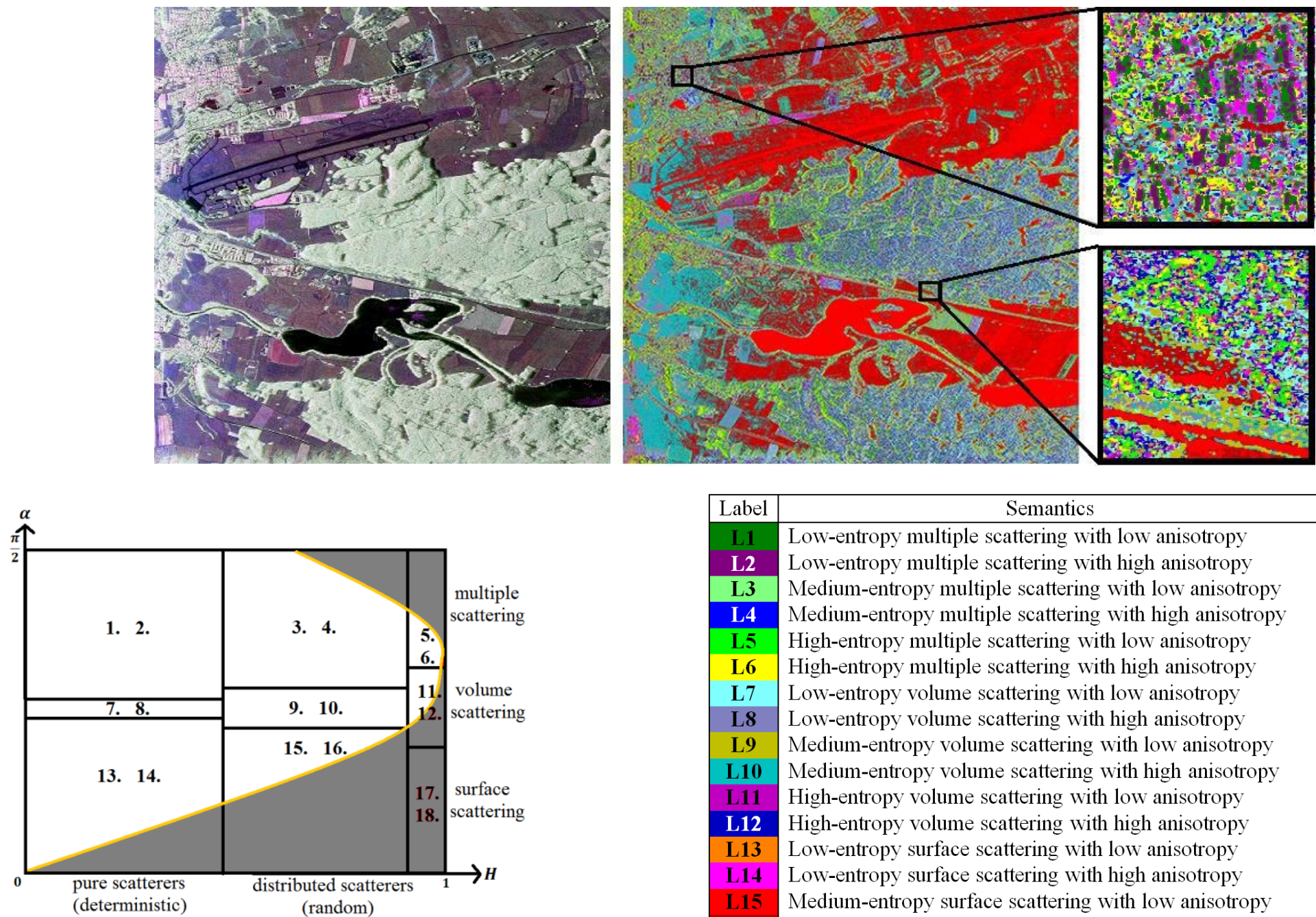

\begin{tabular}{|c|l|}
\hline Label & \multicolumn{1}{|c|}{ Semantics } \\
\hline L1 & Low-entropy multiple scattering with low anisotropy \\
L2 & Low-entropy multiple scattering with high anisotropy \\
\hline L3 & Medium-entropy multiple scattering with low anisotropy \\
\hline L4 & Medium-entropy multiple scattering with high anisotropy \\
\hline L5 & High-entropy multiple scattering with low anisotropy \\
L6 & High-entropy multiple scattering with high anisotropy \\
L7 & Low-entropy volume scattering with low anisotropy \\
L8 & Low-entropy volume scattering with high anisotropy \\
L9 & Medium-entropy volume scattering with low anisotropy \\
L10 & Medium-entropy volume scattering with high anisotropy \\
L11 & High-entropy volume scattering with low anisotropy \\
L12 & High-entropy volume scattering with high anisotropy \\
\hline L13 & Low-entropy surface scattering with low anisotropy \\
L14 & Low-entropy surface scattering with high anisotropy \\
L15 & Medium-entropy surface scattering with low anisotropy \\
L16 & Medium-entropy surface scattering with high anisotropy \\
\hline
\end{tabular}

R Tănase, R Bahmanyar, G Schwarz, M Datcu

Discovery of Semantic Relationships in PolSAR Images Using Latent Dirichlet Allocation

IEEE Geoscience and Remote Sensing Letters 2017
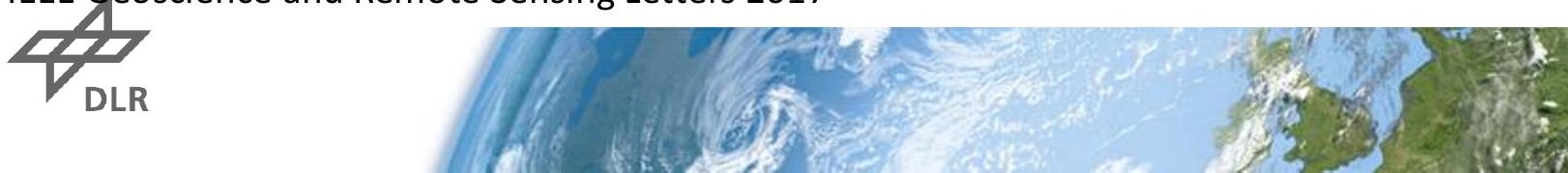


\section{High-level semantics - LDA topics for PolSAR L-Band}

$$
p\left(w_{d n} \mid a, B\right)=\int p\left(\theta_{d} \mid a\right)\left[\sum_{z_{d n}} p\left(z_{d n} \mid \theta_{d}\right) p\left(w_{d n} \mid z_{d n}, B\right)\right] d \theta_{d}
$$
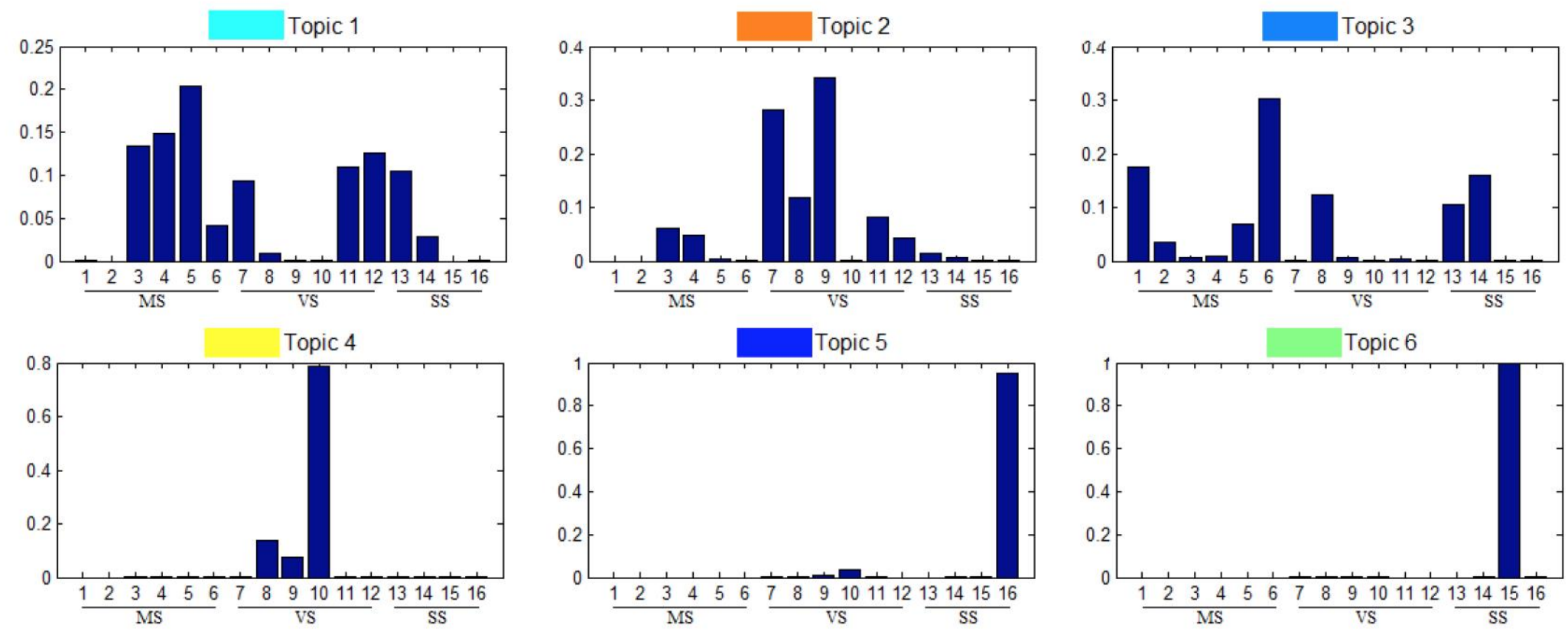

\begin{tabular}{|c|l|l|}
\hline Topic & \multicolumn{1}{|c|}{ Semantics } & \multicolumn{1}{c|}{ Semantic rule } \\
\hline T1 & Woody vegetation & $53 \% \mathrm{MS}+34 \% \mathrm{VS}+13 \% \mathrm{SS}$ \\
T2 & Mixed woody vegetation \& shrubs & $12 \% \mathrm{MS}+86 \% \mathrm{VS}+02 \% \mathrm{SS}$ \\
T3 & Artificial, man-made structures & $60 \% \mathrm{MS}+14 \% \mathrm{VS}+26 \% \mathrm{SS}$ \\
T4 & Herbaceous vegetation & $00 \% \mathrm{MS}+100 \% \mathrm{VS}+00 \% \mathrm{SS}$ \\
\hline T5 & Smooth surface & $00 \% \mathrm{MS}+05 \% \mathrm{VS}+95 \% \mathrm{SS}$ \\
T6 & Specular surface & $00 \% \mathrm{MS}+00 \% \mathrm{VS}+100 \% \mathrm{SS}$ \\
\hline
\end{tabular}
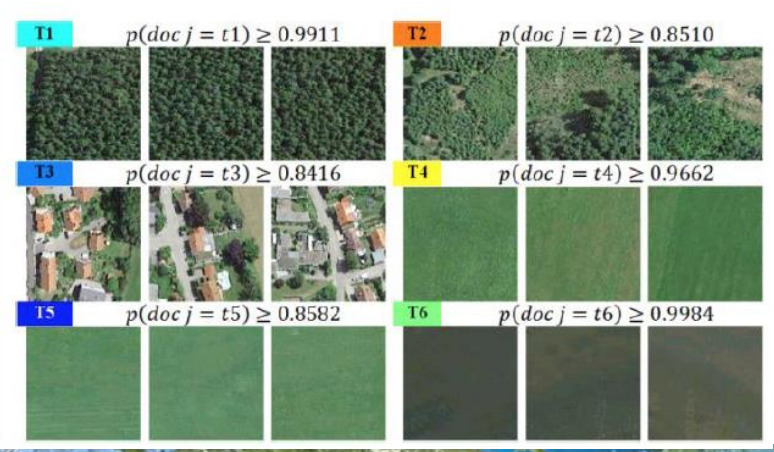


\section{Trends}

- Enforce the market/business of Al and Data Analytics in EO

- Grow multi-disciplinary and disruptive thinking HR

- Apply Al where it is needed and works such to obtain more than $20 \%$ better

- Target to implement solutions for the big, required, but unsolved yet technologies for global and long term EO market:

- Joint multi-mission and multi-source data valorization

- Satellite Image Time Series valorization

- Multisource model assimilation and physical parameters inversion

- Predictive models and forecast Earth dynamic

- Quantum technologies

- Elaborate an overall EO intelligence for the system of systems:

- DATA - SENSOR - MISSION - ARCHITCTURE 


\section{Threats}

- DNN: in 2019 more than $\mathbf{5 0 0}$ papers/month

- Research is often wasted effort

- ML faces a deep reproducibility crisis

- Training data is as important as the learning algorithm

- ML finds any pattern in data, it may be irrelevant

- We need the actual patterns of the Earth processes

- Big EO Data accentuate the crisis

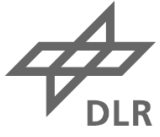




\section{References}

- Dumitru, C.O.; Schwarz, G.; Castel, F.; Lorenzo, J.; Datcu, M.; Artificial Intelligence Data Science Methodology for Earth Observation, in Advanced Analytics and Artificial Intelligence Applications, InTech Publishing, London, UK, September 2019, pp. 75-97, DOI: http://dx.doi.org/10.5772/intechopen.86886

- Tong, X.Y.; Xia, G.S.; Hu, F.; Zhong, Y.; Datcu, M.; Liangpei Zhang Exploiting Deep Features for Remote Sensing Image Retrieval: A Systematic Investigation, IEEE Transactions on Big Data 2019, Early Access Article.

- Bahmanyar, R.; Espinoza-Molina, D.; Datcu, M.; Multisensor Earth Observation Image Classification Based on a Multimodal Latent Dirichlet Allocation Model, IEEE Geoscience and Remote Sensing Letters, vol. 15, issue 3, pp. 459-63, 2018. URL: https://ieeexplore.ieee.org/abstract/document/8278834

- Dumitru, C.O; Schwarz, G.; Datcu, M.; SAR Image Land Cover Datasets for Classification Benchmarking of Temporal Changes, IEEE Journal of Selected Topics in Applied Earth Observations and Remote Sensing, vol. 11, no. 5, pp. 1571-92, 2018, IEEE ISSN 1939-1404, URL: https://ieeexplore.ieee.org/abstract/document/8303669

- Murillo Montes de Oca, A. ; Bahmanyar, R.; Nistor, N.; Datcu, M., Earth Observation Image Semantic Bias: A Collaborative User Annotation Approach, IEEE Journal of Selected Topics in Applied Earth Observations and Remote Sensing, vol. 10, no. 6, pp. 2462 - 2477, 2017, DOI: 10.1109/JSTARS.2017.2697003, URL: https://ieeexplore.ieee.org/abstract/document/7926346

- Tănase, R.; Bahmanyar, R.; Schwarz, G.; Datcu, M., Discovery of Semantic Relationships in PolSAR Images Using Latent Dirichlet Allocation, IEEE Geoscience and Remote Sensing Letters, vol. 14, no. 2, pp. 237 - 241, 2017, DOI: 10.1109/LGRS.2016.2636663, URL: http://ieeexplore.ieee.org/document/7797191/

- Dumitru, C. O.; Schwarz, G.; Datcu, M., Land Cover Semantic Annotation Derived from High-Resolution SAR Images, in IEEE Journal of Selected Topics in Applied Earth Observations and Remote Sensing, vol. 9, no. 6, pp, 2215 - 2232, 2016, DOI: 10.1109/JSTARS.2016.2549557, URL: http://ieeexplore.ieee.org/document/7464254/

- Marmanis, D.; Datcu, M.; Esch, T.; Stilla, U., "Deep Learning Earth Observation Classification Using ImageNet Pretrained Networks," in Geoscience and Remote Sensing Letters, IEEE , vol.13, no.1, pp.105-109, Jan. 2016, doi: 10.1109/LGRS.2015.2499239

- EOLib project; URL: http://wiki.services.eoportal.org/tiki-index.php?page=EOLIB+Project

- ECOPOTENTIAL project; URL: https://www.ecopotential-project.eu/

- CANDELA project; URL: http://candela-h2020.eu/

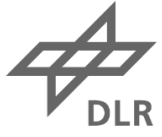

